\title{
Niñez y migración: propuesta cualitativa desde dibujos temáticos y narraciones
}

\section{Childhood and migration: a qualitative proposal based on thematic drawings and narratives}

Dayana Luna-Reyes

Santos Noé Herrera-Mijangos

Jorge Gonzalo Escobar Torres

Universidad Autónoma del Estado de Hidalgo

\section{Resumen}

El presente trabajo tiene como objetivo describir el dispositivo metodológico llevado a cabo a partir del proyecto de investigación colectiva: Percepción de la migración en niños y niñas en municipios con migración internacional en cinco estados de la República Mexicana. El objetivo central fue analizar la experiencia sobre la migración en niños y niñas con padres o madres migrantes en las regiones objeto de estudio. En el caso de este artículo, nos centraremos únicamente en la descripción de la estrategia metodológica que construimos para abordar las percepciones de las y los niños participantes; dicha estrategia está integrada por siete fases: 1. Preparación e inserción al trabajo de campo; 2. Torbellino de ideas; 3. Cuestionario; 4. Dibujo temático; 5. Narración; 6. Espacio grupal para la recuperación de la experiencia de niños y niñas; y 7. Recuperación de experiencia de investigadores. Cada una de las fases son descritas, con hincapié en las técnicas e instrumentos utilizados, las formas de sistematización y análisis de datos. Para finalizar, se presentan tanto un análisis de nuestro dispositivo metodológico como algunas alternativas para mejorarlo y adaptarlo de acuerdo con las necesidades de niñas y niños con familiares migrantes.

Palabras clave: migración, niños y niñas, dibujo temático, narraciones, metodologías cualitativas. docentes.

\footnotetext{
Nota del autor

Dayana Luna Reyes, Área Académica de Psicología, Instituto de Ciencias de la Salud, Universidad Autónoma del Estado de Hidalgo,(UAEH).

Santos Noé Herrera Mijangos, Área Académica de Psicología, Instituto de Ciencias de la Salud, UAEH.

Jorge Gonzalo Escobar Torres, Área Académica de Psicología, Instituto de Ciencias de la Salud, UAEH.

La correspondencia en relación con este artículo debe dirigirse a Dayana Luna Reyes, Área Académica de Psicología, Instituto de Ciencias de la Salud, UAEH, Circuito ex-Hacienda La Concepción, s/n, Carretera Pachuca-Actopan. C. P. 42160, San Agustín Tlaxiaca, Hidalgo, México.

Correo electrónico: dayis2902@gmail.com
} 


\begin{abstract}
The objective of this paper is to describe the methodological device employed as part of the collective research project titled Perception of migration in children in municipalities with international migration in five states of Mexican Republic. The main objective of this project was to analyze the experience of migration in children with migrant parents in the regions under study. In the case of this article, we will focus only on the description of the methodological strategy we constructed to address the perceptions of the participating children; this strategy is composed of seven phases: 1 . Preparation and insertion to the field work; 2. Brainstorming; 3. Questionnaire; 4. Thematic drawing; 5. Narration; 6. Group space for the recovery of the children's experience; and 7. Recovery of experience of researchers. We describe each of the phases, emphasizing the techniques and instruments used, the forms of systematization and data analysis. And finally we present both an analysis of our methodological device and some alternatives to improve it and adapt it according to the needs of children with migrant relatives.
\end{abstract}

Keywords: migration, children, thematic drawing, narrations, qualitative methodologies.

Como hemos comentado en el resumen de este trabajo, en el caso de este artículo, nos centraremos en la descripción y discusión del dispositivo metodológico construido para la indagación de la percepción de niñas y niños sobre la migración. Es importante mencionar que aquí presentamos algunas generalidades de dicho dispositivo, con base en nuestra experiencia durante estas investigaciones $\mathrm{y}$ con la pretensión de resaltar los elementos homogéneos o aquellos en los cuales coincidimos los diversos equipos de investigación de cada uno de los estados participantes; sin embargo, no aspiramos a generalizar el desarrollo y la forma de aplicación del dibujo temático, ya que como bien sabemos la investigación cualitativa es, en sí misma, un arte (Taylor \& Bogdan, 1992), el cual está atravesado tanto por las formas creativas individuales y grupales que desarrolla cada equipo de investigación, así como las vicisitudes y condiciones del trabajo de campo a las que se enfrentan las y los investigadores en su quehacer cotidiano.

De esta forma, el texto pretende recuperar los procesos metodológicos que nos han sido útiles para la exploración de las percepciones sobre la migración de niños y niñas a través de la descripción más o menos detallada del trabajo de campo desarrollado, además de proporcionar una guía metodológica que considera tanto aspectos de aplicación de algunas técnicas como la formación/capacitación del equipo de investigación, las formas de inserción en el campo, el desarrollo y aplicación del dibujo temático, así como la recuperación de la experiencia de los y las niños participantes como del propio equipo de investigación. 
Al final del texto, presentamos tanto un análisis de nuestro dispositivo metodológico como algunas alternativas para mejorarlo $\mathrm{y}$ adaptarlo de acuerdo con las necesidades de niñas y niños con familiares migrantes. Parte de estos resultados, así como una discusión somera del proceso metodológico se presentaron en la mesa "Dimensiones psicosociales de la migración" dentro del Tercer Congreso Latinoamericano de Ciencias Sociales realizado en la ciudad de Zacatecas, cuyos trabajos fueron publicados en el libro Reestructuración y vigencia del modelo neoliberal en América Latina, en el capítulo: "Percepciones de la migración en niños y niñas con familiares migrantes en el estado de Hidalgo, México".

Antes de describir nuestra estrategia metodológica, nos interesa presentar de manera general algunos de los objetivos que orientaron nuestro proceso de investigación conjunta, llevada a cabo desde la participación de cinco equipos de investigación en diferentes estados de la República Mexicana, a saber: Zacatecas, Yucatán, Michoacán, Hidalgo y Jalisco. Especificamos, también, que este proyecto nació en el seno de la Cátedra en Psicología Julieta Heres Pulido, del Consorcio de Universidades Mexicanas (CUMex).

El objetivo general de nuestro proyecto era analizar la experiencia sobre la migración en niños y niñas con padres o madres migrantes en las regiones objeto de estudio; a su vez, los objetivos específicos fueron:
1. Conocer y analizar la percepción de niños y niñas miembros de familias migrantes de las regiones objeto de estudio.

2. Analizar el significado que le dan a la migración los niños y niñas con padres y madres migrantes.

3. Identificar y analizar las experiencias con la migración de niños y niñas miembros de familias migrantes de las regiones objeto de estudio, considerando, en su caso, la influencia e impacto de ésta en la vida familiar.

4. Conocer y analizar los aspectos afectivoemocionales de niños y niñas de familias migrantes de las regiones objeto de estudio.

5. Proponer una estrategia metodológica para el estudio y la intervención del fenómeno de la migración y su relación con la salud mental.

Dados los objetivos de este estudio, concordamos con Vargas (1994), quien conceptualiza la percepción como un proceso cognitivo que consiste en el reconocimiento, la interpretación y significación en la elaboración de juicios relativos a las sensaciones obtenidas desde el ambiente físico y social. De acuerdo con la misma autora, en la percepción intervienen procesos psíquicos como el aprendizaje, la memoria y la simbolización.

De ahí la importancia de las percepciones como eje conceptual de nuestro estudio, ya que 
queríamos acercarnos a dichas significaciones, construidas a partir de los procesos de aprendizaje en la vida cotidiana de las niñas y los niños con familiares migrantes; para tal fin, se identificaron aspectos de su memoria que, posiblemente, contienen las historias narradas de sus familiares y se entrelazan con las simbolizaciones que ellas y ellos recrean al dibujar y narrar sus propias experiencias en torno a la migración.

De esta forma y de acuerdo a Barriga (1985, como se citó en Osorio, 2018), el estudio de las percepciones se constituye en una vía para conocer la realidad del sujeto, quien se conecta con el mundo a través de las sensaciones, organizándolas mediante la interpretación de los estímulos recibidos.

\section{Del dispositivo metodológico}

Para el desarrollo de esta investigación nos planteamos la realización de un estudio transversal de tipo exploratorio y descriptivo, para el cual necesitábamos un enfoque metodológico que nos permitiera acercarnos con mayor profundidad a la vida cotidiana de las niñas y niños con familiares migrantes, así como obtener elementos que nos acercaran a la compresión de los significados que ellos le dan al proceso migratorio, junto con la posibilidad de explorar los afectos y emociones involucrados en su experiencia de vida, como lo es la ausencia de los familiares migrantes.

De acuerdo a Cazau (2006), el que un estudio sea exploratorio o descriptivo dependerá del enfoque que el investigador busque darle a su estudio; puede ser inicialmente exploratorio para posteriormente centrarse en la descripción de los procesos involucrados. Desde esta perspectiva, optamos por generar una estrategia metodológica que nos permitiera, a la vez de explorar los aspectos psicosociales centrales en la percepción de las niñas y los niños acerca de la migración, describir sus experiencias, sus procesos afectivo - emocionales, las formas en que se vivían las dinámicas al interior de sus familias, así como las significaciones que construyen en torno a la migración de sus familiares.

De acuerdo a Monje (2011), los diseños cualitativos se caracterizan por ser poco fijos y modificables; se inicia desde un punto de referencia para enseguida ajustarse de acuerdo con las características del campo. Debido a lo anterior, optamos por la construcción de un dispositivo, cuyo eje central fuera de enfoque cualitativo, ya que para nosotros dicho enfoque, además de ser flexible, con diseño emergente (Sandoval, 2002) y creativo, posibilita la utilización de técnicas que permiten recoger las voces desde los propios participantes, sus experiencias y significaciones (Delgado \& Gutiérrez, 1994). Por consiguiente, consideramos así como informantes principales a las niñas y los niños con familiares migrantes.

De esta forma, caracterizamos a nuestros informantes clave (Rodríguez, Gil \& García, 1996) y delineamos los siguientes criterios de inclusión: 
- Ser niño o niña de entre 8 y 12 años de edad.

- Cursar los últimos grados de la formación primaria $\left(4^{\circ}, 5^{\circ}\right.$ o $6^{\circ}$ grado).

- Cursar en una escuela primaria pública.

- Vivir en una comunidad con índice de emigración considerable.

- Tener algún familiar cercano que haya emigrado.

De acuerdo con estas características, nuestro trabajo de campo se desarrolló con la participación de un total de 664 niños y niñas, distribuidos como se muestra en la tabla 1, por cada equipo de investigación en cada uno de los estados participantes.

A continuación, presentamos el dispositivo o estrategia metodológica que implementamos, recordando que, en este caso, se trata de una propuesta metodológica integrada a partir de la experiencia de los cinco equipos de investigación, misma que se centra en la elaboración de dibujos temáticos referentes a la migración, desarrollados de manera libre, tanto por niños como por niñas, quienes también tuvieron la posibilidad de explicar su dibujo o narrar una pequeña historia sobre el mismo.

Entonces, la estrategia metodológica para el trabajo de campo contempló un proceso integrado por varias fases:

- Fase 1. Preparación e inserción al trabajo de campo.

- Fase 2. Torbellino de ideas.

- Fase 3. Cuestionario.

- Fase 4. Dibujo temático.

- Fase 5. Narraciones.

- Fase 6. Espacio grupal para la recuperación de la experiencia de niños y niñas.

- Fase 7. Recuperación de experiencia de investigadores.

Tabla 1

Distribución de niños y niñas participantes por estado

\begin{tabular}{lccc}
\hline Estado & Niños & Niñas & Total \\
\hline Hidalgo & 59 & 66 & 125 \\
Jalisco & 107 & 67 & 174 \\
Michoacán & 56 & 60 & 116 \\
Yucatán & 71 & 92 & 163 \\
Zacatecas & 38 & 48 & 186 \\
Total & 331 & 333 & 664 \\
\hline
\end{tabular}


A continuación, describiremos cada una de estas fases.

\section{Fase 1. Preparación e inserción al trabajo de campo.}

Para el desarrollo de esta fase es importante centrarse en tres aspectos:

a. La capacitación del equipo de aplicadores e investigación. En este aspecto, se hizo necesario integrar un equipo de investigación, que en muchos de los casos se integró por docentes investigadores y estudiantes. El proceso de capacitación permitió tanto socializar los objetivos de la investigación como homogeneizar los conocimientos teóricos y metodológicos respecto a las técnicas que se aplicarían durante el trabajo de campo: unificar y clarificar entre todos los miembros del equipo el proceso de aplicación, incluyendo las formas de dar instrucciones, las fases de la actividad y capacitación respecto a las formas de expresión y comunicación para la interacción con niños y niñas. Esta capacitación también permitió la integración grupal del equipo de investigación, lo cual generó un clima de trabajo basado en la confianza, el respeto y la ayuda mutua. Es importante mencionar que en alguna de las sesiones de capacitación se habló acerca de las ansiedades y miedos que los integrantes del equipo estaban experimentando a raíz del trabajo con el tema y con los participantes.

b. La gestión de los permisos institucionales para realizar la investigación. Este aspecto se refiere a realizar algunas visitas a los diversos escenarios donde se contemplaba llevar a cabo el trabajo de campo. De esta forma, acudimos a las diferentes escuelas primarias y nos entrevistamos con los directivos de las mismas para solicitar su autorización para la realización de la investigación. Se elaboraron y entregaron los oficios y demás documentación requerida para obtener los permisos, tanto para trabajar con los niños y niñas, como del uso de los espacios para la aplicación de las diversas técnicas. En estas primeras visitas con directivos, fue necesario solicitar datos respecto al número de grupos y la cantidad de alumnos en cada uno para poder organizar de antemano las parejas de aplicadores y la integración de paquetes con materiales necesarios. De igual manera, se realizaron algunos recorridos por los municipios o poblaciones donde estaban instaladas las primarias públicas que se visitarían, con el fin de familiarizarnos con los espacios y formas de llegada.

c. La gestión de los recursos técnicos y materiales necesarios para realizar la investigación. Considerando este rubro, preparamos con antelación al trabajo de campo propiamente dicho, los materiales que utilizaríamos para la aplicación de las técnicas, de modo que fueran suficientes y estuvieran organizados. Esto contempló la integración de paquetes por pareja de aplicadores que contenían: lápices, gomas, sacapuntas, hojas blancas, lápices de colores, cuadernos de campo, etc. En algunas ocasiones, también preparamos cámaras fotográficas y/o celulares 
con memorias listas para la toma de fotografías en las primarias que así lo autorizaron, siempre y cuando no se tomaran las caras de las y los niños para preservar su anonimato. Por otra parte, fue necesario elaborar cartas de agradecimiento dirigidas a docentes y personal directivo.

\section{Fase 2. Torbellino de ideas.}

Una vez en el campo y al interior de cada aula, fue necesario presentarnos ante los niños y niñas con nuestros nombres de pila, procedencia y solicitándoles su apoyo para participar en diversas actividades relacionadas con la migración. Algunos estudiantes nuestros de Psicología, quienes también integraron los equipos de aplicadores, comentaban: "somos alumnos y alumnas de Psicología de la UAEH y queremos que nos ayuden elaborando unos dibujos".

Se les planteaba que su participación era voluntaria y anónima; no se les pedirían nombres ni se les tomarían fotos.

Una vez que las y los niños accedían a participar, implementábamos inicialmente un espacio de charla grupal en el cual explorábamos lo que ellos entendían por migración. Enseguida, se les preguntó grupalmente "¿qué entienden por migración?", $y$, a manera de torbellino de ideas, las niñas y los niños iban participando de manera voluntaria, mencionando ejemplos tanto de la migración de animales como de personas y familias. Se recolectaban las diversas opiniones, tratando de focalizar la temática de interés, de tal manera que las niñas y los niños se centraran en elucidar en conjunto lo que entendían por migración.

De acuerdo a De Prado (2001), el torbellino de ideas (brainstorming, concepto acuñado por Osborn en 1938), es una técnica de actividad creativa que consiste en la expresión libre y espontánea de ideas, relacionadas con un tema o un problema o, en su caso, las respuestas libres a una pregunta, teniendo como regla la no crítica, represión, reproche o rechazo a ninguna opinión.

Siguiendo a De Prado (2001), el torbellino de ideas es una técnica participativa que promueve la aportación, ya sea individual o grupal, de manera flexible, espontánea, libre y diversa; se aplica en un breve período, que va desde algunos minutos hasta un cuarto de hora y cuya finalidad es la creación de una mayor cantidad y calidad de ideas en torno a un tema.

De esta forma, el torbellino de ideas se puede constituir en una estrategia útil para el trabajo con niños y niñas, ya que permite la obtención de opiniones diversas y espontáneas de los mismos sobre un tema en una menor cantidad de tiempo, a la vez que permite acercarnos a la idea colectiva, en este caso, respecto a la migración de los familiares.

Fue importante que el equipo de investigación contara con un cuaderno de notas o diario de investigación en el cual anotar comentarios, actitudes, estados anímicos, posturas, interrelaciones, formas de participación y de atención de los niños y las niñas durante el proceso de aplicación, con la finalidad tanto de complementar las percepciones de la migración 
como contextualizar y dar formas posibles, referenciales, desde donde leer los dibujos realizados.

En este ejercicio, nos percatamos de la similitud de sus respuestas, y de que, además, conocían lo que es migración, incluso, algunos se referían directamente a la migración de algún familiar o conocido.

\section{Fase 3. Cuestionario.}

Una vez focalizado el tema de la migración con las niñas y los niños, les repartimos a cada uno una hoja que tenía impreso un cuestionario con preguntas abiertas, cuyo fin era la identificación de las características socioculturales generales de los niños y niñas que elaborarían los dibujos. Dicho cuestionario consta de once preguntas que se contestaron de manera individual y anónima:

1. ¿Eres niño o niña?

2. ¿Cuántos años tienes?

3. ¿En qué año vas en la escuela?

4. ¿De dónde eres o dónde naciste?

5. ¿Tienes familiares que han emigrado a otro lugar?

6. ¿Quién o quiénes de tus familiares han emigrado?

7. ¿A qué lugar se fue o se fueron?

8. Para ti, ¿qué es la migración?

9. Para ti, ¿es buena o mala la migración?

10. ¿Por qué?

El cuestionario tiene una onceava pregunta, sin embargo, para ese momento de la aplicación sólo se les pidió contestar hasta la pregunta 10 .
Fue importante que los aplicadores estuvieran atentos a las dudas que las niñas y los niños tuvieran respecto a esta parte de la actividad, para aclararlas y motivar su contestación.

Con el fin de sistematizar la información, se elaboraron matrices de concentrado de datos numéricos como cantidad de niños y niñas, edades, grados, etc., para su posterior análisis a través de la identificación de medidas de tendencia central; además de un tratamiento cualitativo de las respuestas de las preguntas 8,9 y 10 , con la elaboración de tablas comparativas entre datos cualitativos.

\section{Fase 4. Dibujo temático.}

Considerando que el presente trabajo se inscribe en una propuesta metodológica de tipo cualitativo, posiblemente uno de los aspectos más centrales de nuestra propuesta sea la utilización del dibujo temático como vía para la exploración de las percepciones que, de manera gráfica, tienen las niñas y los niños respecto a la migración.

Como recordaremos, en nuestros criterios de inclusión, retomamos los dibujos de niños y niñas que cursaban los últimos tres años de la instrucción primaria, lo cual nos sitúa en un rango de edad de entre los 8 y los 12 años. Para Wallon (1999, como se citó en Aguilera, 2009), esta etapa de desarrollo es la del realismo, y es donde "... el dibujo no es la representación del objeto en sí, sino que es la representación de la experiencia que el niño recoge en estos grupos [refiriéndose a los grupos sociales importantes 
en la vida del niño]" (p. 15). En nuestro caso, los grupos o colectivos presentes en la vida de estas niñas y niños fueron la familia, el grupo de amigos en la escuela y la comunidad en general donde viven; de ahí la importancia del dibujo como una vía de acceso a sus representaciones respecto a la migración.

Ojeda García (2014) menciona que el dibujo puede ser una vía para el estudio de las representaciones simbólicas de los niños y niñas en su vida diaria, se constituye en una forma de conocer su "mundo interno", facilitando la identificación de elementos para la planeación de un trabajo subsecuente, que en nuestro caso estaría enfocado en un proceso de atención de niños y niñas con familiares migrantes y sus comunidades.

De acuerdo a Wallon, Cambier y Engelhart (1999), el dibujo temático implica la representación de conocimientos y la elección de qué se va a representar y desde qué sistema de codificación. Por su naturaleza, un dibujo de este tipo apela a la capacidad del dibujante (un niño o niña en este caso) para situarse con respecto a lo real y a lo imaginario sobre el fenómeno.

Siguiendo a Arroyo (2009), los infantes dicen muchas cosas de sí mismos a través del dibujo. El dibujo puede ser, en algunos casos, un termómetro del estado de ánimo, ya que puede traducir lo que la o el niño siente, piensa, desea o lo que a él o ella le inquieta, le hace alegre o triste.
Existen numerosos estudios (ChacónGordillo \& Sánchez-Ruiz, 2009, 2013; JiménezYáñez \& Mancinas-Chávez, 2009), que utilizan el dibujo como una técnica de trabajo con infantes para acceder a las formas que tienen de relacionar sus experiencias con la cultura y sus procesos sociales; permite obtener información acerca de los participantes, sus procesos grupales y sobre su entorno.

Según Rovetta (2017), dentro de la metodología cualitativa se ha recurrido en las últimas dos décadas a la utilización de lo que se llaman métodos visuales, en conjugación con técnicas tradicionales como entrevistas y grupos de discusión. A esta combinación podemos llamarla técnicas de elucidación (Meo \& Dabenigno, 2011): permiten introducir técnicas visuales como detonantes para la elaboración individual o grupal de las formas en que las personas participantes representan sus experiencias, lo que piensan y sienten respecto a ellas y cómo los contextos socioculturales les dan contenido.

Siguiendo a Rovetta (2017), estas técnicas de elucidación han sido utilizadas en diversos estudios cualitativos con niños y niñas, sobre todo en el ámbito anglosajón, empezando a trabajarse en Iberoamérica.

Es así que, de acuerdo con nuestro proceso investigativo, en esta fase retomamos la aplicación del dibujo temático; cuando las niñas y los niños terminaron de contestar el cuestionario, se les pidió que voltearan sus hojas, se les proporcionaron lápices de 
colores, sacapuntas, gomas y lápices de grafito, indicándoles que dibujaran de manera individual lo que era para ellos la migración.

El tiempo para elaborar el dibujo temático fue libre, sin embargo, el tiempo requerido para tal tarea oscilaba entre media hora y 45 minutos. En algunas ocasiones a algún infante no le gustaba cómo había iniciado su dibujo o se equivocaba, entonces se le proporcionaba otra hoja. En caso de que algunos elementos del dibujo no se entendieran, no eran claros o explícitos, se le preguntaba qué era y se anotaba en la parte posterior de la hoja.

En caso de que algún niño o niña no quisiera dibujar, se intentaba convencerlo indicándole que no se trataba de un concurso de dibujo, ni tampoco de ver quién dibujaba mejor, que había libertad y para nosotros era muy importante saber cómo dibujaba la migración. En este aspecto, algunos equipos de investigación optaron por realizar una entrevista individual a manera de conversación con las y los niños que no quisieron elaborar su dibujo. Cabe destacar que se presentaron muy pocos casos, empero, fue necesario respetar la decisión del participante, con quien se trabajaron las preguntas del cuestionario y una exploración verbal sobre lo que opinaba respecto a la migración.

Lo que pudimos observar en esta fase de la aplicación es que tanto niñas como niños sacaron de sus mochilas lápices de colores, los compartían entre sí; se centraban en su dibujo de manera individual y si tenían dudas preguntaban a los aplicadores.
Durante la elaboración del dibujo se mostraron entusiasmados, emocionados, activos, sonrientes, dedicados, contentos, intrigados; las y los niños se sintieron libres. Involucrados en la tarea de dibujar la migración.

Para la sistematización, se procedió a identificar cada uno de los dibujos con una clave y un folio, relacionado con la escuela, el turno (matutino o vespertino), el grado escolar y si se trataba de niño o de niña participante. Se escaneó cada uno de los dibujos, identificando los archivos con su clave y número de folio.

La técnica del dibujo aplicada no se trata de un dibujo proyectivo que requiera un análisis de tipo clínico, sino que lo importante para su interpretación, son los contenidos manifiestos del dibujo y su composición integral.

De esta forma, el estudio de los dibujos se basó en identificar sus contenidos manifiestos, así como los elementos más frecuentes $\mathrm{y}$ representativos en los dibujos de los niños y niñas, dejando de lado aspectos como los colores y las formas, ya que se trata de un análisis de representaciones gráficas de la experiencia migrante y los elementos significativos que los niños y las niñas asocian con dicho proceso.

Inicialmente, se identificaron cuatro categorías de análisis de los dibujos:

1. La migración (referente a los miembros de la familia que han emigrado).

2. Los motivos (económicos, riñas, mudanza o gusto).

3. Los efectos (económicos, emocionales). 
4. Los símbolos (vehículos, frontera, bandera, camino, etcétera).

Más tarde, estas categorías bien pudieron ser categorías transversales de los siguientes ejes:

1. Migración y procesos socioemocionales.

2. Migración e identidades sociales.

3. Perspectivas del proceso migratorio desde niñas y niños.

4. Narrativa y migración desde las niñas y los niños.

Tanto estos ejes como las categorías han servido de líneas analíticas de los resultados de cada uno de los equipos de investigación de cada estado donde se realizó paralelamente la investigación.

\section{Fase 5. Narraciones.}

De acuerdo a Pava-Ripoll (2015), las narraciones son versiones del mundo y develan las formas en que se organiza la experiencia humana. Pava-Ripoll (2015) también realiza un estudio considerable respecto a las diversas propuestas respecto al uso de las narraciones como estrategia para investigar los procesos mediante los cuales los participantes construyen identidades y modos de significar su mundo a partir de sus narraciones en torno a sus experiencias de vida.

El uso de las narraciones es otra de las técnicas centrales de nuestro proceso investigativo. Focalizado desde la metodología cualitativa, tratamos de recoger las voces de las niñas y los niños a través de la narración y/o descripción de sus dibujos. Las narraciones pudieron ser parte de las historias contadas en sus círculos sociales próximos, como parte de su historia familiar o comunitaria respecto a la suerte de los familiares o habitantes en su proceso de emigrar. Las narraciones también recogieron sus emociones y sentimientos; los sucesos y trayectorias; las esperanzas y deseos de las niñas y los niños sobre sus familiares.

Una vez que terminaban su dibujo, se les pedía de favor que voltearan su hoja del lado de las preguntas, y leyeran la pregunta número 11: "Describe tu dibujo o cuéntanos una historia, relato o cuento sobre tu dibujo". Esta actividad fue también de elaboración individual. La actividad podía tardar de 15 a 20 minutos.

Cuando terminaban su narración, se verificaba que tuviera los datos solicitados: dibujo e historia. En caso de que alguna figura de su dibujo no fuera entendible se le pedía que la describiera o nombrara anotándola en el reverso de su dibujo. De igual forma se hacía cuando alguna palabra o letra de su narración no era entendible.

Para la sistematización de las narraciones, procedimos a capturar dichas narraciones de manera íntegra en la misma matriz de los datos sociodemográficos de los niños y las niñas, respetando faltas de ortografía, estilos de redacción, jergas y expresiones idiomáticas. Para el análisis del contenido de estas narraciones (Colle, 2011), procedimos de la misma forma en que se analizaron los dibujos, es decir, 
identificando ejes y categorías manifestadas en las narraciones.

\section{Fase 6. Espacio grupal para la recuperación de la experiencia de niños y niñas.}

A partir de nuestra experiencia en la aplicación de los dibujos y las narraciones, observamos que, en varias ocasiones, los niños y niñas trataban de mostrar sus dibujos y comentar respecto a sus historias con sus compañeros de al lado; en otras ocasiones, había dificultad por parte de ellos para dibujar o, en su caso, para narrar la historia, por esto, decidimos implementar un espacio a manera de reflexión grupal respecto a la actividad realizada. Un espacio para poder trabajar algunas emociones, pensamientos $\mathrm{y}$ opiniones de las niñas y los niños, generadas a partir de la creación de los dibujos y las historias.

Dicho espacio consistió en que los niños y niñas, en caso de que así lo decidieran, mostraran sus dibujos y los describieran al resto del grupo, contaran su relato a todos, hablaran sobre su experiencia respecto a la migración de algún familiar y/o comentaran sobre lo que les significó realizar la actividad.

Para posibilitar lo anterior, se realizó una charla grupal con participaciones voluntarias de las niñas y los niños con preguntas abiertas, por ejemplo:

- ¿Qué dibujo hicieron?

- ¿Qué representa su dibujo?

- ¿Qué historia realizaron respecto a su dibujo?

- ¿Qué les han contado sus familiares sobre la migración?
- ¿Alguien quiere contar alguna experiencia?

- ¿A quiénes de ustedes les gustaría migrar?, ¿a dónde?, ¿por qué?

- ¿Cómo se sintieron con la actividad? ¿Les gustó o no?, ¿por qué?

Esta última actividad fue realizada a manera de cierre de la sesión, recuperando los estados anímicos de los participantes y enfatizando la socialización y la importancia de compartir sus pensamientos y sentimientos, así como revalorar sus dibujos, sus historias y experiencias.

Posterior a esta charla, se les pedía a las niñas y niños que nos proporcionaran sus dibujos; en algunas ocasiones, ellos querían quedarse con su dibujo, lo cual resolvimos tomando una foto legible de la actividad realizada en cada una de las páginas de la hoja y rotulándola con los datos referenciales del participante ( $\sin$ nombres).

También, en algunas ocasiones de la aplicación, tuvimos la oportunidad de llevar dulces que se les entregaban a los niños y niñas al final de la sesión en señal de agradecimiento por su participación. Sin embargo, fue recomendable no mencionarlos ni exhibirlos ni antes ni durante la actividad, sino hasta el final, cuando los niños y niñas habían ya entregado sus dibujos, de tal forma que este factor no tuviera injerencia en el desarrollo de la sesión.

Para finalizar, se les agradecía a las niñas y niños su tiempo y participación en nuestra investigación; así también a los docentes y directivos. 
Para la recuperación de las participaciones voluntarias de las niñas y de los niños, recurrimos asimismo a las anotaciones en el diario de investigación o en el cuaderno de notas de campo, recordando que estos datos servirían de contexto y complemento para la lectura de la información arrojada por los dibujos y las narraciones.

Durante esta última actividad recogimos comentarios como:

"Mi papá me abandonó cuando tenía un año" (niña de 9 años).

"Mi mamá se fue cuando tenía tres años, yo me quedé con mi abuelita. Pero ya regresó mi mamá hace como un año" (niña de 10 años).

"Lluvia nació allá, sabe inglés y de allá la trajeron para acá. Ya nos contó su experiencia" (niño de 9 años).

A la pregunta sobre quiénes se irían a Estados Unidos, cerca de $80 \%$, en cada uno de los grupos, respondía que afirmativamente.

\section{Fase 7. Recuperación de experiencia de apli- cadores e investigadores.}

Con la finalidad de recuperar algunos datos a partir del equipo de investigación, se les pidió que, además del diario de investigación, contestaran una bitácora de recuperación de experiencia, la cual constó de doce preguntas abiertas, relacionadas con las formas de organización del trabajo de campo, las impresiones sobre la comunidad y la escuela donde se aplicaron tanto los cuestionarios como los dibujos de las niñas y los niños, aspectos que llamaron su atención respecto a los dibujos, sobre sus respuestas al cuestionario y demás comentarios sobre la actividad realizada.

Dicha bitácora fue contestada de manera individual por cada uno de los integrantes del equipo, quienes realizamos el trabajo de campo. Las preguntas que contenía la bitácora fueron:

1. De ser la primera vez que visitaste el municipio, ¿qué impresión tuviste al conocerlo?

2. ¿Qué te pareció la escuela?

3. ¿Qué opinas de la organización y distribución de tareas, previos al llegar a la escuela?

4. Del grupo que te tocó coordinar, aproximadamente ¿cuántos alumnos eran en el grupo?

a. ¿Mayoría hombres o mujeres?

5. ¿Cuál era el ambiente o atmósfera del grupo cuando...?

a. Llegaste.

b. Durante la aplicación.

c. Al irte.

6. ¿Hubo algún problema con la impartición de las instrucciones de la actividad?, ¿en qué fase?

7. ¿Existió algún detalle significativo con las respuestas de los niños y niñas a las preguntas planteadas?

8. De los dibujos que llegaste a observar, ¿alguno(s) llamó tu atención sobre los demás?, ¿por qué? 
9. ¿Qué tipo de detalles en los dibujos de los niños y niñas se te hicieron más significativos?

10. ¿Llegaste a leer alguna de las historias?, ¿algo llamó tu atención sobre ellas?

11. Al final, en la retroalimentación grupal, ¿hubo comentarios, frases, actitudes o conductas que llamaran tu atención?

12. Si tienes algún comentario o aportación en cuanto a la organización, desarrollo del proyecto o sobre la experiencia misma, favor de compartirla.

Esta bitácora nos permitió recuperar la experiencia vivida de cada uno de los integrantes del equipo de investigación, es decir, conocer el impacto en nosotros como aplicadores, nuestras premisas y miedos para el trabajo de campo.

Algunos de los comentarios recuperados se transcriben a continuación:

"Me impresionó la historia de los dos hermanos (¿el tío y el padre?). Es una historia tan sombría, tan negativa... se lee el dolor muy fácilmente" (aplicador estudiante).

"Un niño comentó que los policías mataban a las personas, que cruzaban a Estados Unidos, que hacían explotar los autos, que los mexicanos debían de esconderse para que no los mataran, que sus familias los extrañaban... pero que era bonito pensar que regresarían a su casa con dinero para trabajar los terrenos" (aplicadora estudiante).

"Me llamó la atención una de las historias, que aunque fue muy breve, me llamó la atención su sinceridad, en la cual manifiesta muy claramente que su experiencia migratoria fue muy desafortunada y se sentía triste por ello" (aplicadora, investigadora).

"La directora de una de las primarias comentó que hay niños y niñas que caminan varias horas para llegar a la escuela. Comentó que esa primaria es una escuela 'muestra' ya que a esa escuela llegan niños y niñas de varias comunidades cercanas" (aplicador, investigador).

"Nosotros preocupados porque los niños no fueran a entender el término de 'migración', ... 'y si no saben qué es la migración ¿tenemos que explicarles?'. Y cuál fue la sorpresa al dar las instrucciones iniciales, percatarnos que todas y todos los niños conocían el concepto y sus campos semánticos a partir de la experiencia familiar. Lo habían vivido de manera cercana al interior de sus familias y/o comunidades" (aplicador, estudiante).

"Me pregunto si su historia es un cuento y si tiene alguna relación con sus historias personales-familiares" (aplicador, investigador).

Pudimos visualizar que existe poca relación entre los estudiantes y docentes universitarios con la población en general, había poco conocimiento respecto a sus dinámicas sociodemográficas como de sus procesos de vulnerabilidad psicosocial. De ahí la angustia generada en los aplicadores respecto a si los niños y las niñas conocían lo que era la migración.

Por otra parte, las experiencias de vida de ambos colectivos participantes (los aplicadores 
y los infantes), pareciera que estaban en direcciones paralelas, sin guardar ningún tipo de relación. El desconocimiento de los aplicadores sobres las realidades sentidas y expresadas a partir de los dibujos y sus narrativas generaban asombro y extrañeza al equipo de investigadores. La realidad cruda de las familias en proceso de migración constituye un mundo diferente al que los libros, tan socorridos por nosotros los universitarios, no tienen acceso y menos desde las miradas de los niños y de las niñas.

\section{Consideraciones finales}

Como pudimos observar, el dispositivo metodológico que construimos a partir de nuestra experiencia de campo se centra en la implementación de una sesión integrada por varias fases, en cada una de ellas se desarrolla una técnica que es secuencial y cuenta tanto con estrategias para su implementación como para la sistematización y análisis de datos. De acuerdo también con nuestra experiencia, la sesión tiene una duración de entre hora y treinta minutos a dos horas.

Rovetta (2017) recupera algunos comentarios proporcionados por diferentes autores respecto a las ventajas que tiene el uso de métodos visuales en el estudio con niños y niñas:

1. Motiva la participación activa de los infantes (Barter \& Renold, 2000, citado por Rovetta, 2017).

2. Proporcionan una ventaja comunicativa para los participantes con poca intervención de quien investiga (Mitchell, 2006, citado por Rovetta, 2017).

3. Proporciona mayor tiempo a los investigadores para establecer relaciones más cercanas con los participantes (Bagnoli, 2009, citado por Rovetta, 2017).

4. Permiten la implementación simultánea de múltiples técnicas, así como el análisis de datos de manera complementaria (Moskal, 2010, citado por Rovetta, 2017).

5. Una forma alternativa de estudio no basada únicamente en lo verbal o en lo numérico (Copeland \& Agosto, 2012, citado por Rovetta, 2017).

En el caso nuestro, consideramos estas cinco ventajas, así como la facilidad de aplicación; una vez que se tienen los permisos necesarios, es fácil llevarla a cabo, en poco tiempo y con grupos grandes de participantes cautivos (en tanto que se encuentran inmersos en una institución escolar). Por otra parte, la calidad de los datos cualitativos obtenidos a través de los dibujos temáticos, nos permite identificar los elementos presentes en su vida cotidiana, además de los aspectos que tienen relación con las formas de representar su experiencia de vida con la migración.

Posiblemente una desventaja sea que no a todo infante le gusta dibujar o que el sólo hecho de plantear las actividades genere ansiedad en los participantes, al tener una vivencia muy 
cercana y reciente de algún familiar migrante. Esto último implicaría buscar alternativas de trabajo, identificando posibles espacios para los niños y niñas que pudieran presentar dichas ansiedades. Una propuesta es que se trabajen lugares personalizados, a manera de entrevistas individuales.

Por otra parte, el dibujo temático es una técnica que logra involucrar a niños y niñas en la tarea, según nuestra experiencia, quienes se muestran motivados, interesados y entusiasmados tanto por elaborar sus dibujos como por crear sus historias. Se trata de una actividad lúdica que convoca a la exploración de datos a través de la creación artística.

En el caso de las narraciones, de acuerdo a Richardson y St. Pierre, (2005, citados por García-Huidobro, 2016), priorizan el relato, la expresión oral y escrita; además de permitir reconstruir lo vivido de una manera más natural (Denzin \& Lincoln, 2011, citados por GarcíaHuidobro, 2016).

Según Mowling, Brock y Hastie (2006, citado por Moragón-Alcañiz \& Martínez-Bello, 2016), en un estudio donde también utilizaron el dibujo y las narraciones como técnicas de investigación, se percataron de la importancia de las narraciones para la interpretación de los dibujos.

De esta forma, el dibujo temático junto con la narración de historias se constituyen en vías metodológicas para explorar el mundo familiar de las niñas y los niños participantes, sus percepciones respecto a la migración y cómo estas vivencias generan emociones $\mathrm{y}$ afectos en ellos, configurando identidades sociales. La propuesta de este trabajo bien puede ser una vía de aproximación al mundo subjetivo de niños y niñas; permite explorar no sólo las vivencias experimentadas al tener un familiar migrante, sino también sus percepciones sobre la misma y sus expectativas sobre la vida propia.

Optamos por dispositivos metodológicos de tipo cualitativo que nos faciliten profundizar y hacercontacto con las propias vivencias dey desde los participantes; estrategias metodológicas que estén adaptadas a sus realidades y dinámicas, a su vez que permitan la exploración de sus vidas cotidianas para identificar sus necesidades sentidas y expresadas para posteriores intervenciones. El dispositivo metodológico también tendría que responder al tipo de tema desde el cual se está trabajando, partiendo de la complejidad de la problemática y cuidando, desde un aspecto ético, que el propio dispositivo metodológico no violente sus derechos humanos y atienda sus necesidades específicas, al tiempo que evite la revictimización, al colocarlos en lugares vulnerables o su invisibilización con abordajes estereotipados que no recojan de viva voz sus propuestas.

Los procesos migratorios forman parte de la vida cotidiana de las niñas y de los niños; se encuentra en las vidas y dinámicas de sus familias, en su repertorio semántico, con el cual se relacionan con el mundo y también forma parte de sus expectativas de vida a manera de ideales y como vías para mejorar su calidad de 
vida. Migración y trabajo, desde la perspectiva de las niñas y los niños, tienen una relación directamente proporcional, ocupan un eje central en sus vidas y, a la vez, dicha relación convive con la experiencia de sufrimiento, separación, ausencias y muerte. En estos casos, la migración como opción desesperada ante la pobreza, ya es violencia social (Luna-Reyes \& Rodríguez, 2015, p. 14).

Es necesario partir de la escucha de la voz de tantas niñas y niños que experimentan las ausencias del familiar migrante, haciendo un esfuerzo por poner en tela de juicio la visión adultocéntrica (Ballestín, 2009) que tanto hemos fortalecido desde la academia y desde los programas de atención social.

La propuesta puede servir, asimismo, como estrategia de investigación, de diagnóstico $\mathrm{y}$ de intervención psicosocial. Puede ser un antecedente para identificar procesos problemáticos tanto en niños y niñas, familias y comunidades con la intencionalidad de generar programas de atención a familias en situación de migración, de tal forma que favorezca el desarrollo integral de los niños, niñas y sus familias. Consideramos la necesidad de generar políticas públicas y sociales que sostengan programas de prevención y atención, basados en la promoción de la salud integral y los derechos humanos.

Se hace urgente la atención de las niñas y los niños con familiares migrantes; la atención de las parejas de las y los migrantes; el apoyo comunitario de los cuidadores de los hijos e hijas de migrantes; el fortalecimiento de redes comunitarias para el trabajo con quienes regresan y también, con aquellos que se quedan.

\section{Referencias}

Aguilera, A. B. (2009). El dibujo infantil. Revista Digital Ciencia y Didáctica, (8), 9-17.

Arroyo, M. V. (2009). El color en el dibujo del niño. Revista Digital Ciencia y Didáctica, (23), 4-12.

Ballestín, B. (2009). La observación participante en primaria: ¿Un juego de niños? Dificultades y oportunidades de acceso a los mundos infantiles. Revista de Antropología Iberoamericana, 4(2), 229-244. Recuperado de https://www.redalyc.org/articulo.oa?id= 62312915004

Cazau, P. (2006). Introducción a la investigación en ciencias sociales. Buenos Aires: RundiNuskín.

Chacón-Gordillo, P. y Sánchez-Ruiz, J. (2009). La estructura familiar de Los Simpsons (C a través del dibujo infantil. Revista mexicana de investigación educativa, 14(43), 11291154. Recuperado de http://www.scielo. org.mx/scielo.php?script=sci_arttext\&pid $=\mathrm{S} 1405-66662009000400007$

Chacón-Gordillo, P. y Sánchez-Ruiz, J. (2013). La estructura familiar dibujada por los propios niños/as. Una aproximación al dibujo como mediador social en las relaciones sistémicas familiares. Revista de bellas artes: revista de artes plásticas, estética, diseño e imagen, 
(11), 159-180. Recuperado de http://dialnet. unirioja.es/servlet/articulo?codigo $=4401287$ Colle, R. (2011). El Análisis de contenido de las comunicaciones. 3. Ejemplos de aplicaciones. Tenerife: Sociedad Latina de Comunicación Social. Recuperado de http:// www.revistalatinacs.org/067/cuadernos/13_ Colle_interior.pdf

Delgado, J. M. y Gutiérrez, J. (Ed.). (1994). Métodos y técnicas cualitativas de investigación en ciencias sociales. Madrid: Síntesis.

De Prado, D. (2001). Torbellino de Ideas: Por una educación participativa y creativa. Santiago de Compostela, España: Mieubook. Recuperado de http://educreate.iacat.com/ Biblioteca_prado/31.Dpd.TI.Educacion.pdf

García-Huidobro, R. (2016). La narrativa como método desencadenante y producción teórica en la investigación cualitativa. Revista de Metodología de Ciencias Sociales, 34(2), 155-178.

Jiménez-Yáñez, C. y Mancinas-Chávez, R. (2009). Semiótica del dibujo infantil: Una aproximación latinoamericana sobre la influencia de la televisión en los niños: Casos de estudios en ciudades de Chile, El Salvador y México. Arte, individuo y sociedad, (21), 151-164. Recuperado de http://revistas.ucm. es/index.php/ARIS/article/view/6608

Luna-Reyes, D. y Rodríguez, V. (2015). Percepciones de la migración en niños $\mathrm{y}$ niñas con familiares migrantes en el estado de Hidalgo, México. En R. Ibarra, E.
Bueno, R. Ibarra y J. L. Hernández (Eds.), Reestructuración y vigencia del modelo neoliberal en América Latina. México: UAZTaberna Libraria.

Meo, A. y Dabenigno, V. (2011). Imágenes que revelan sentidos: Ventajas y desventajas de la entrevista de foto-elucidación en un estudio sobre jóvenes y escuela media en la ciudad de Buenos Aires. Empiria. Revista de Metodología de Ciencias Sociales, (22), 1342.

Monje, C. (2011). Metodología de la investigación cuantitativa y cualitativa. Guía didáctica. Colombia: Programa de comunicación social y periodismo NEIVA.

Moragón-Alcañiz, F. y Martínez-Bello, V. (2016). Juego de niñas y juego de niños: Estudio sobre la representación del juego infantil a través del dibujo. Revista Educación, 40(1), 1-17.

Ojeda, A., Bailón, C. y García, G. (2014). El dibujo como expresión del mundo interno del migrante. México: Universidad Iberoamericana.

Osorio, C. (2018). La percepción en la publicidad vs. la percepción en la psicología. Colección Académica de Ciencias Sociales, 5(1), 5059. Recuperado de https://revistas.upb. edu.co/index.php/cienciassociales/article/ view/8023/7315.

Pava-Ripoll, N. (2015). Narrativas conversacionales con familias y docentes de niños y niñas con discapacidad: un aporte 
metodológico. Interdisciplinaria, 32(2), 203222.

Rodríguez, G., Gil, J. y García, E. (1996). Metodología de la investigación cualitativa. Granada: Aljibe.

Rovetta, A. (2017). "Si me dieran un billete de avión...": recurriendo a la elucidación gráfica en entrevistas con menores de edad. Revista de Metodología en Ciencias Sociales, 36(1), 63-87.

Sandoval, C. A. (2002). Investigación cualitativa. Bogotá: ICFES.

Taylor, S. J. y Bogdan, R. (1992). Introducción a los métodos cualitativos de investigación. Barcelona: Paidós.

Vargas, L. M. (1994). Sobre el concepto de percepción. Alteridades, 4(8), 47-53. Recuperado de https://www.redalyc.org/ pdf/747/74711353004.pdf

Wallon, P., Cambier, A. y Engelhart, D. (1999). El dibujo del niño. Madrid: Siglo XXI.

Recibido: 14/03/2019

Revisado: 18/06/2019

Aceptado: 25/06/2019 